\title{
Smartphone Application for Self Blood Glucose Monitoring \& Disease Management- A Case Report
}

\author{
Dr. Moshe Kamar MD. \\ Department of Acute Care, \\ Wolfson Medical Center \\ 62 Halochamim St. \\ Holon Israel \\ 972543301308 \\ moshe@mydario.com
}

\section{Categories and Subject Descriptors}

J. COMPUTER APPLICATIONS

\section{J.3 LIFE AND MEDICAL SCIENCES}

Subject descriptor: Medical information systems

\section{General Terms}

Performance, Measurement, Management

\section{Keywords}

Glucose Meter, Glucose Monitor, Diabetes, T1 Diabetes, Type 1 Diabetes, Diabetes Performance, HbA1C, Diabetes Compliance, Blood Sugar, Blood Glucose, Diabetes SelfManagement

\begin{abstract}
Self-management of blood sugar glucose, diet and control of daily activities are crucial in overall management of type 2 diabetes mellitus (DM). In order to better adjust insulin type and dosage, educate for better diet patients are seldom requested to conduct a diary of their glucose measurements and meals. In today's era of smartphone widespread use, it has been suggested that using mobile technology for self-blood glucose measurement together with the ability to automatically capture other factors important to control DM, may be an important method for enhancing disease selfmanagement.

The Dario ${ }^{\mathrm{TM}}$ device and mobile application, is a self-blood glucose monitoring device using smartphone interface. The application accumulates data of measurements, insulin intake, meals and physical activity. Users are also members of an electronic DM support group.

We present a case of a 55 y.o. type $2 \mathrm{DM}$ patient who reported using the device over a year period. The patient baseline $\mathrm{HbAlc}$ before using the Dario program was 7.4. He started using the Dario device and application and over a period of one year he managed to gradually reduce $\mathrm{HbA} 1 \mathrm{c}$ to 6.2 by keeping his glucose measurements in range from $20 \%$ a week to $100 \%$.
\end{abstract}

\section{CONCLUSIONS}

The use of mobile technologies together with 
multidisciplinary approach may revolutionize DM disease management.

\section{INTRODUCTION}

Type 2 Diabetes Mellitus (DM) is now considered an epidemic in both children and adults in the United States, it affects 18.2 million people or $6.3 \%$ of the population. ${ }^{1,2}$ Estimated health care expenditures associated with diabetes include $\$ 91.8$ billion in direct and $\$ 39.8$ billion in indirect costs as of 2002. The cause of such high morbidity, mortality, and costs can be mostly attributed to long-term, chronic diabetes-related complications, such as renal disease, coronary heart disease, blindness, and neuropathy. ${ }^{3}$ It has been long advocated that aggressive control of patients' blood sugar levels, together with lifestyle practices and adherence to prescribed interventions lowers morbidity and mortality associated with the disease. According to the American Diabetes Association, diabetes self-management is an integral component of diabetes care. Improved glycemic control (hemoglobin A1c <7\%) has been shown to minimize microvascular complications by decreasing the rates of nephropathy, retinopathy, neuropathy, and cardiovascular disease. $^{4}$

Disease control is a daily struggle in all aspects of lifestyle, diet, physical activity, mental status, sleep, adherence to medication regiment etc. In an era when smartphone are owned by almost everyone and social networking (i.e. Facebook, Twitter, WhatsApp...) is present and active on every smartphone, the ability is created to monitor DM patients glucose levels, lifestyle, alert them of out of range results, automatic remind them of medication and other activities that are required. Results can be sent to their treating physicians for better understanding and holistic care. The use of social networks connects people with the disease, caregivers and other professionals providing "support groups" and motivating channels.

We report a case of a type 2 DM patient who used a Dario ${ }^{\mathrm{TM}}$ (Labstyle Innovations Ltd, Israel) type self-blood glucose monitoring device (SBGM) who reported over the social media network, great results in disease management.

Dario's Diabetes Management Solution consists of a proprietary software component in the format of an iOS (iPhone Operating System) and Android based application where the user's data is saved and managed in one healthy lifestyle management system. Dario sets a new standard for diabetes personalization by leveraging social network, medical alerts, insights and pattern recognition techniques in order to provide accurate and proactive analysis and recommendations for a PWD (People with Diabetes). The Dario management application supports full diabetes lifecycle - blood glucose monitoring, food intake, insulin dosage and physical activity while allowing the PWD take a proactive role in managing his diabetes. In addition, the Dario application incorporates basic features such as a measurement $\log$ (when connecting the Dario glucose meter), data capturing, personal trend graphs, sharing and alert features. Moreover, the Dario management system can be accessed through the Dario website portal where PWD can observe in more details their history and trends and share their reports with their physicians, caregivers and loved ones. ${ }^{5}$

\section{CASE REPORT}

P.B.* is a 55 y.o. type 2 insulin dependent DM patient diagnosed in 2006 and was put on short acting insulin. At the same time he was diagnosed also to have mild hypertension. After several years of uncontrolled disease with numerous episodes of severe hypo and hypers he was recommended to use a smartphone based glucose monitor in an attempt to better understand the confounders of his disease. He started using the Dario Diabetes Management Solution in June 2014 and was instructed to test his blood glucose four times a day. Baseline HbA1c when entering the program was $7.4 \%$.

Together with a dietician P.B. was given a diet specifying the amount of carbohydrates he needs to consume daily which he recorded through his Dario App. Insulin type and treatment regimen were entered baseline visit and updated when treatment was changed. Patient was instructed to have 4 measurements a day, and enter his meal contents every time he had one . He could also enter episodes of stress, illness, sleep disorders, travels and other situations which are out of his routine daily activities. After each meal carbs are automatically calculated, Dario enabled the patient to receive the recommended dosage of insulin based on all the recorded measurements.

Patient was also entered into a designated online social community where he could share his experiences with other users, get support from professional and technical personnel as to how to manage the disease and utilize his Dario in order to benefit his condition. 
For each glucose measurement device would categorize measurement as falling "in range" or "out of range" and feedback the patient on his progress by showing percentage of measurements falling in range over time.

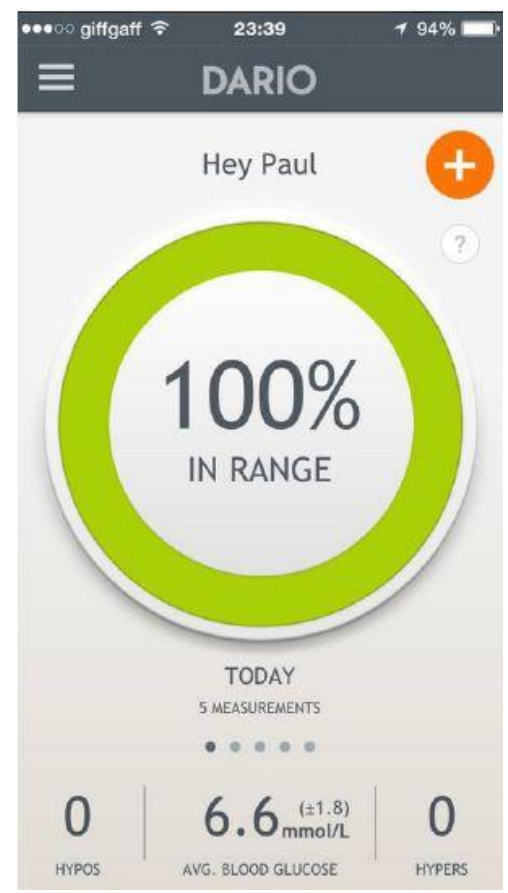

P.B. Dario “in range' screen shot

Before each monthly visit to the clinic cumulative data graphically displayed was sent to the clinic for us to assess his progress and tailor treatment accordingly.

\section{RESULTS}

At the commencement of use, the patient's weekly average glucose measurements were $9.4 \mathrm{mmol} / \mathrm{l}(169 \mathrm{mg} / \mathrm{dl})$. At the end of the year weekly average glucose measurements were down to $5.7 \mathrm{mmol} / \mathrm{l}(102 \mathrm{mg} / \mathrm{dl})$ a decrease of $60 \%$ (figure 1$)$. Initial "in range" results were in $20 \%$ of the measurements which came up to $100 \%$ at the end of the year.

These results were reflected in his laboratory $\mathrm{HbA} 1 \mathrm{c}$ results as well as in device calculated A1C, demonstrating an improvements of $83 \%$ with reductions from 7.4 to 6.2 . (figure 2)

The patient reported overall great satisfaction from the device and application: "The app is so simple to use, take a blood sample, enter what food you are eating and it gives you the carb content then press the symbol and you get the correct dosage of insulin, I love it takes all the guess work out of carb counting. I love the display on the app it's so simple to read and vivid. I have the app on my iPhone iPad and iPod I love it that I can use all these to do a test and all my results will sync" (taken from P.B.'s social network postings).

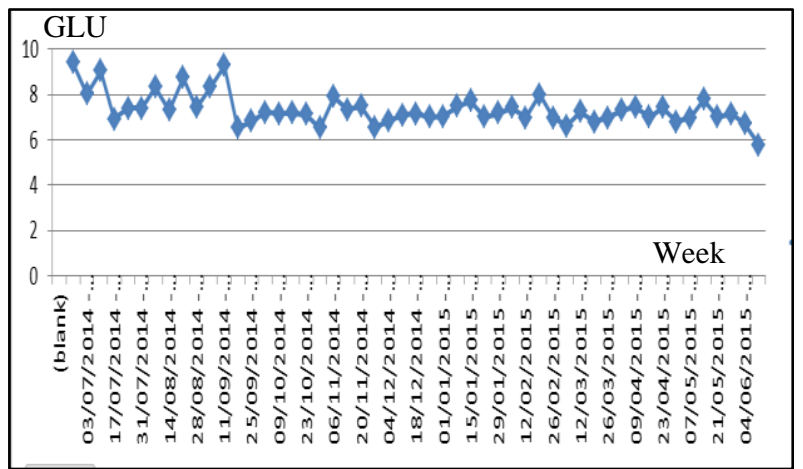

Figure 1: showing gradual decrease of up to $60 \%$ from baseline measurements in blood glucose values ( $\mathrm{mmol} / \mathrm{l})$

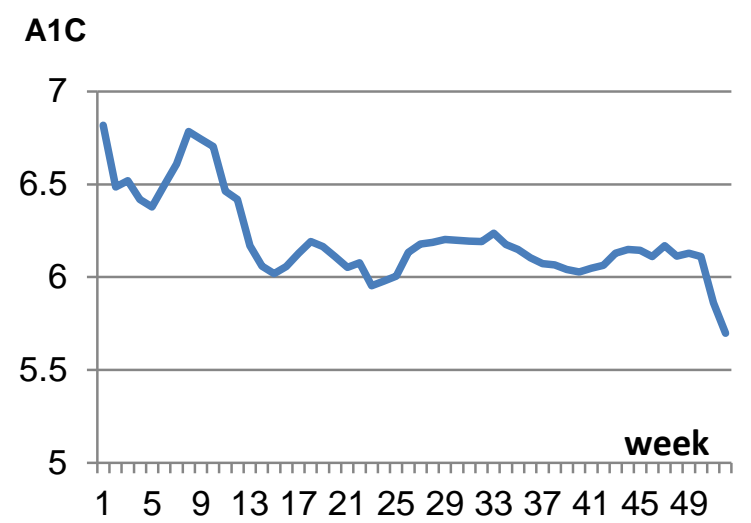

Figure 2 - showing $83 \%$ decrease in HbA1C over 52 weeks period

\section{DISCUSSION}

Thriving with diabetes is a daily battle in which every patient is required to self-manage and control multiple variables. In order to tailor treatments and assist in construction of "the DM routine" patients are typically required to manage a diary. There is a constant "wild goose chase" to understand one's food content, activities and other variables affecting blood glucose levels. It has been shown previously how integrated daily use is more likely if the self-management components are offered in a mobile phone app, and electronic diaries are thought to improve self-management. ${ }^{6,7}$ Certainly the patient should be the primary "user" of that data, but even the most motivated patient needs a little guidance, a few reminders, and a lot of education to be maximally effective. The medical community is unprepared to collect or analyze all data 
requested. The logical step would be to streamline the process, automate as much as possible, and help each patient become as self-efficacious as possible. Management of chronic disease, including diabetes, has passed the point in which quarterly visits with a quick review of daily home measurements is adequate to maintain optimal health. Data collected should be used to be an effective tool. Minimal action resulting from home monitoring destroys the motivation necessary to continue the collection of that data. Certainly the patient should be the primary "user" of that data, but even the most motivated patient needs a little guidance, a few reminders, and a lot of education to be maximally effective. The medical community is unprepared to collect or analyze all data requested. The logical step would be to streamline the process, automate as much as possible, and help each patient become as self-efficacious as possible.

The wide spread use of smartphones, internet and social networks open up the possibility for multimodal approach. From the patient side automated features reduce some burden of entering data, other data can be entered in an easy and friendly way. Furthermore the device can notify the patient of activities/actions needed (i.e. medications, BG testing, doctor appointments etc.), Compiling all data in real time assists the patient in determining insulin dosage and it can also alert the patient of significant changes which require special attention or action.

The use of smartphone technology allows for a freer interchange between patients and the health care team. Physicians are capable of understanding the data over time and effectively focus on events/ periods affecting patient glucose balance. ${ }^{8}$

Studies of mobile phone-based interventions have had varied success in improving self -management and glycemic control in individuals with diabetes. ${ }^{9,10}$ One explanation is that mobile phones are a platform, not a solution in itself, and interventions vary widely. A major gap in the literature is the lack of behavioral models to explain how these interventions improve diabetes self-management. ${ }^{11}$ Reviews of diabetes self- management interventions studies of mobile phone applications to date have been largely theoretical. The prevailing theoretical assumption is that mobile phone-based interventions lead to behavior change through prompts and conditioning. ${ }^{12}$ However, barriers to self-management are complex, and it is unlikely that sustained behavior change can be observed through conditioning alone. ${ }^{13}$
This case report demonstrates the ability of new technologies to have significant impact on patient disease self-management. The availability, low cost, prevalence use of smartphone apps' and interfaces increased the patient's ability to control his disease and gradually for the first time take charge. Together with the device, support group and health care provider he has managed to produce a routine that promoted him to maintain his blood glucose to a desired range, lower his HbA1c and above all self-satisfaction from his freedom, to carry the device everywhere, getting real time information and feedback on the process.

\section{CONCLUSIONS}

Smartphone based technologies for controlling diabetes, have a promising role in the holistic and multidisciplinary of disease management.

*Patient P.B. has given complete consent to utilize his measurements and information. 


\section{REFERENCES}

1. Centers for Disease Control and Prevention. National diabetes fact sheet: general information and national estimates on diabetes in the United States, 2003. Atlanta (GA):

2. U.S. Department of Health and Human Services, Centers for Disease Control and Prevention; 2003.

3. Hogan P, Dall T, Nikolov P; American Diabetes Association.Economic costs of diabetes in the US in 2002. Diabetes Care.2003;26:917-32.

4. American Diabetes Association. Standards of medical care for patients with diabetes mellitus. Diabetes Care. 2003;26:S33-S50

5. Vuong AM, Huber JC, Bolin JN, Ory MG, Moudouni DM, Helduser J, et al. Factors affecting acceptability and usability of technological approaches to diabetes self-management: a case study. Diabetes Technol Ther 2012 Dec;14(12):1178-1182

6. LabStyle Innovations; Dario Diabetes Management Solution. www.mydario.com

7. Holmen H1, Torbjørnsen A, Wahl AK, Jenum AK, Småstuen $\quad$ MC, Arsand E, Ribu L. A Mobile Health Intervention for SelfManagement and Lifestyle Change for Persons With Type 2 Diabetes, Part 2:One-Year Results From the Norwegian Randomized Controlled Trial RENE WING HEALTH. JMIR Mhealth Uhealth. 2014 Dec $11 ; 2(4): e 57$.
8. Malasanos T. ANALYSIS: mobile phones integrated into diabetes management: a logical progression. J Diabetes Sci Technol. 2008 Jan;2(1):154-5

9. Krishna S, Boren SA. Diabetes self-management care via cell phone: a systematic review. J Diabetes Sci Technol. 2008; 2:509-517

10. Holtz B, Lauckner C. Diabetes management via mobile phones: a systematic review. Telemed J E Health. 2012; 18:175-184

11. Riley WT, Rivera DE, Atienza AA, Nilsen W, Allison SM, Mermelstein R. Health behavior models in the age of mobile interventions: are our theories up to the task? Transl Behav Med. 2011; $1: 53-71$

12. Dick JJ, Nundy S, Solomon MC, Bishop KN, Chin $\mathrm{MH}$, Peek ME. Feasibility and usability of a text message-based program for diabetes selfmanagement in an urban African-American population. J Diabetes Sci Technol. 2011; 5:12461254

13. Nundy S1, Dick JJ, Solomon MC, Peek ME. Developing a behavioral model for mobile phonebased diabetes interventions. Patient Educ Couns. 2013 Jan;90(1):125-32. 